\title{
Return to play following metacarpal fractures in football players
}

\author{
Brian E. Etier ${ }^{1}$ - Anthony J. Scillia ${ }^{2}$ - Darin D. Tessier ${ }^{2} \cdot$ Kyle T. Aune $^{2}$. \\ Benton A. Emblom ${ }^{2,3}$ - Jeffery R. Dugas ${ }^{2,3}$ - E. Lyle Cain ${ }^{2,3}$
}

Published online: 21 May 2015

(C) American Association for Hand Surgery 2015

\begin{abstract}
Background Metacarpal fractures are common in football players and lead to time away from competition, but current operative treatment data is limited. The purpose of this study was to examine demographics and outcomes of football players who underwent operative fixation for metacarpal fractures.

Methods Retrospective review from 2009 to 2012 of any football player surgically treated at one institution for a metacarpal fracture. Charts were reviewed for player position, level of competition, mechanism of injury, return to play, postoperative bracing, and re-fracture event. Current information was obtained via phone interviews with the patient and their athletic trainers. Fractures were classified by radiographic analysis.

Results Twenty injuries in ten high school players, nine college players, and one recreational player were identified. The most common injured position was wide receiver (six cases) followed by defensive back (five cases). Most injuries occurred through player-to-player contact (12 cases). The long finger (11 cases) was most commonly involved metacarpal. Two players had multiple metacarpal fractures. The most common location was mid-diaphyseal (15 cases). The mean return to play for all in-season athletes was 6.3 days (range 1-21).
\end{abstract}

Brian E. Etier

betier@uabmc.edu

1 University of Alabama at Birmingham Hospital, 1313 13th Street South, Birmingham, AL 35205, USA

2 American Sports Medicine Institute, 833 St. Vincents Drive, Birmingham, AL 35205, USA

3 Andrews Sports Medicine and Orthopaedics Center, 805 St. Vincents Drive, Birmingham, AL 35205, USA
Protective splints were used for an average of 21 days (range 14-36). All athletes returned to their preinjury level of play without recurrence of fracture or wound complication.

Conclusion Football players who required surgical fixation of a metacarpal fracture demonstrated an efficient return to play, including in-season players with use of protective bracing. Study Design Case series, Level of evidence, IV

Keywords Metacarpal fractures · Football players · Operative $\cdot$ Return to play $\cdot$ Bracing

\section{Introduction}

Metacarpal fractures are a common injury accounting for approximately $18 \%$ of fractures below the elbow and $10 \%$ of all upper extremity fractures $[1,2]$. The mechanism of injury involves direct trauma or fall onto an outstretched hand with a clenched fist [3]. As such, these injuries frequently occur in contact sports such as football, hockey, and wrestling $[10,12]$. Metacarpal fractures are commonly treated nonoperatively with good success using cast or splint immobilization in the intrinsic plus position for 4 weeks. The indications for nonoperative treatment include absence of rotational deformity, no articular step-off, less than $10^{\circ}$ coronal angulation, less than $10^{\circ}$ sagittal angulation in the index and long fingers, less than $20^{\circ}$ sagittal angulation in the ring finger, and less than $30^{\circ}$ sagittal angulation in the small finger [8]. Minimal shortening is accepted in the metacarpal shaft, as each $2 \mathrm{~mm}$ of shortening has been demonstrated to correspond to $7^{\circ}$ of extensor lag [11].

Conservative management of metacarpal fractures has been demonstrated to be an effective treatment option $[6,9]$. Surgical treatment of displaced metacarpal shaft fractures allows for anatomic restoration, fracture stability, and early range of 
motion and rehabilitation, which could also decrease complications such as stiffness and muscle atrophy [7]. This could potentially result in a more expeditious return to play timeline among in-season athletes, and previous studies have reported athletes returning as quickly as 1 week following surgical fixation [3-5]. All athletes in these studies utilized a protective brace when they initially returned to play.

Although prior studies have described return to play protocols among athletes in general, discussions of return to play timelines for individual sports are lacking. To our knowledge, there are no previous studies that specifically address return to play, bracing, or associated complications of operative metacarpal fractures specifically among football players. The purpose of the present study was therefore to review injury characteristics, as well as the results of operative treatment of metacarpal shaft fractures in a cohort of high school and college level football players. Specifically, we assessed (1) patient demographics, (2) injury characteristics, (3) operative procedures, (4) mean length of return to play, and (5) postoperative protective equipment use.

\section{Material and Methods}

Retrospective data was collected for all patients following institutional review board approval. Patients were identified by a computerized search using the current procedural terminology (CPT) code for open treatment of metacarpal fracture. The search was limited to patients who underwent surgical treatment by three surgeons (B.A.E., J.R.D., E.L.C.) at our institution from 2010 to 2012. Surgical indications included a displaced metacarpal fracture with rotational deformity or multiple metacarpal fractures. Forty-six patients with a metacarpal fracture were treated over that 3-year period. Twelve patients did not participate in sports. Thirteen patients were involved in sports other than football, which included basketball, baseball, softball, soccer, martial arts, motor cross, and cheerleading. Twenty-one of the 46 patients participated in football at the collegiate, high school, or recreational level. One high school athlete was lost to follow-up and excluded from the study.

Retrospective chart review was performed to identify player position, level of competition, fracture event and mechanism, return to play, postoperative bracing, and re-fracture event. Completion of data was performed by telephone interview of the athlete and/or the athlete's athletic trainer. Return to play was defined as the number of days between surgical fixation and return to full contact practice or game.

Radiograph assessment, including posteroanterior, lateral, and oblique views of the hand at the time of injury, were reviewed in all patients. These radiographs were used to classify fracture site, anatomic location, and character. Operative reports were reviewed for implant choice.
Data was recorded in an Excel spreadsheet (Microsoft Corporation, Redmond, Washington), which was also used to calculate summary data statistics including in-season return to play as well as for brace time.

\section{Results}

Twenty patients met inclusion criteria for the study. Nine were competing at the collegiate level at the time of injury. Of those, eight were National Collegiate Athletic Association Football Bowl Subdivision athletes and one was a Division III athlete. Ten patients were playing high school football, and one was playing collegiate intramural football. The most commonly treated positions were wide receiver (six players) and defensive back (five players). Three linebackers, three running backs, one offensive lineman, one defensive lineman, and a tight end each sustained a metacarpal shaft fracture that met operative indications. Nine players were injured during practice; eight players were injured during a game. Two players were injured during weight training. One collegiate football player sustained an injury while playing recreational basketball.

Metacarpal fractures most commonly took place with player-to-player contact (12 patients). Two players sustained a fracture following contact with the ground. Four athletes were injured by contact with equipment, getting their hand twisted in a jersey, catching the football, or being stepped on. Two sustained noncontact injuries while lifting weights (Table 1).

The long finger was most commonly fractured, and most fractures were mid-diaphyseal in location. The appearance of short oblique, transverse, and spiral fractures were evenly distributed. Complete fracture site, anatomic location, and character data are presented in (Table 2).

Twelve football players underwent open reduction and internal fixation during football season. Eight of the 12 had surgery less than 2 days after injury. Three of 12 underwent fixation less than 4 days after injury. One patient had fixation 14 days after injury, and following failure of conservative cast treatment. The eight out-of-season fractures underwent operative fixation between 0 and 16 days. Most commonly, operative fixation was carried out using a Synthes (West Chester, PA) 2.4-mm LCP modular mini fragment 4, 5, or 6 hole plate (eight patients). Two patients were stabilized with Synthes 2.0-mm LCP modular mini fragment plate. One patient underwent fixation with Synthes $2.7-\mathrm{mm}$ LCP modular mini fragment plate; one patient had fixation of his metacarpal base fracture with a Synthes 2.4-mm condylar plate. A 2.4-mm F3 Fragment Plating System Plate (Biomet, Inc./Hand Innovations, Miami, FL) was used for fixation of one patient. Six athletes underwent fixation with Biomet Hand Innovations 1.6-mm metacarpal nail. One $2.0-$ or $2.4-\mathrm{mm}$ lag screw 
Table 1 Patient demographics

\begin{tabular}{ll}
\hline & Number/total \\
\hline Competition level & \\
Collegiate & $9 / 20$ \\
High school & $10 / 20$ \\
Recreational & $1 / 20$ \\
Position at injury & \\
Wide receiver & $6 / 20$ \\
Defensive back & $5 / 20$ \\
Linebacker & $3 / 20$ \\
Running back & $3 / 20$ \\
Offensive/defensive lineman & $2 / 20$ \\
Tight end & $1 / 20$ \\
Injury event & \\
Practice & $9 / 20$ \\
Game & $8 / 20$ \\
Off-season workout & $2 / 20$ \\
Recreational basketball & $1 / 20$ \\
Injury mechanism & \\
Player to player contact & $12 / 20$ \\
Player to ground contact & $2 / 20$ \\
Non specific injury & $6 / 20$ \\
\hline
\end{tabular}

accompanied plate fixation in five patients. One patient underwent fixation with three 2.4-mm lag screws only.

The mean return to play for all in-season athletes was 6.3 days (median 5.5 days, range 1-21 days). Return to play for all football players ranged from 1 to 42 days and averaged 16.9 days. The average time to return to play for in-season high school football players was 9.2 days (median 8.5 days;

Table 2 Fracture demographics

\begin{tabular}{ll}
\hline & Number/total \\
\hline Metacarpal & \\
Long finger & $11 / 20$ \\
Small finger & $3 / 20$ \\
Ring finger & $2 / 20$ \\
Index finger & $1 / 20$ \\
Thumb & $1 / 20$ \\
Long and ring finger & $1 / 20$ \\
Index and long finger & $1 / 20$ \\
Location & \\
Mid-diaphyseal & $15 / 20$ \\
Neck & $3 / 20$ \\
Base & $2 / 20$ \\
Character & \\
Transverse & $7 / 20$ \\
Spiral & $7 / 20$ \\
Short oblique & $6 / 20$ \\
\hline
\end{tabular}

range 3-15 days) and 2.8 days (median 3 days; range $1-$ 4 days) for in-season collegiate football players (Table 3 ). One college recreational athlete returned to play without protective equipment at 21 days. All athletes were able to return to their preinjury level of play, and none suffered recurrence of fracture.

All 11 in-season high school and college athletes returned to play with protective equipment in the form of a padded glove, bivalve padded cast, padded club cast, or padded splint. Equipment type varied on football position. Skill positions such as running backs, wide receivers, and defensive backs used padded gloves; offensive lineman and linebackers used padded casts (Table 4 ). The protective equipment was used for an average of 21 days (range 14-36 days).

\section{Discussion}

Following surgical fixation of a metacarpal fracture, in-season high school and college athletes were able to return to play in less than a week, and college football players in less than 3 days. All athletes returned to their preinjury level play or higher without any instance of re-injury. Metacarpal fractures are a common, competition-limiting injury among American football players. To date, however, there remains a paucity of return to play data following operative fixation of this fracture type.

Return to play of athletes following metacarpal fractures has been previously documented. Geissler reported on ten athletes who underwent open reduction and internal fixation of metacarpal fractures. Eight underwent plate fixation, and the other two were stabilized with lag screws. Sport and position played were not included in the study, however. All athletes returned to play within 1 to 2 weeks. One athlete sustained a re-fracture through lag screws at 1 year postoperatively [4]. Geissler and McCraney previously suggested plate fixation of metacarpal fractures in contact athletes who wished to return to play quickly. These authors suggested 2-mm plates with four cortices purchase both proximal and distal to the fracture. With adequate fixation achieved, contact athletes were able to return to play after several weeks wearing a fracture brace [5]. Rettig et al. reported on 56 metacarpal fractures in 53 athletes of various sports. Five football players with fractures underwent open reduction and internal fixation with a return to play of 13.6 days [10]. Fufa and Goldfarb

Table 3 Return to play of in-season athletes

\begin{tabular}{ll}
\hline & Return to play (days) \\
\hline In-season & \\
College & 2.8 \\
High school & 9.17 \\
Combined & 6.27 \\
\hline
\end{tabular}


Table 4 Protective equipment for in-season fractures

\begin{tabular}{lllll}
\hline & Equipment & Level & Return to play (days) & Brace time (days) \\
\hline Position & & & \\
Wide receiver & Padded glove & College & 1 & 21 \\
Wide receiver & Club clast & High school & 15 & 19 \\
Running back & Bivalve cast & College & 4 & 14 \\
Defensive back & Padded glove & College & 4 & 21 \\
Defensive back & Ulnar gutter splint & High school & 3 & 28 \\
Tight end & Orthoplast foam pad & College & 3 & 28 \\
Linebacker & Club cast & High school & 7 & 18 \\
Linebacker & Club cast & High school & 10 & 19 \\
Linebacker & Club cast & College & 2 & 21 \\
Defensive lineman & Bivalve cast & High school & 7 & 36 \\
Offensive lineman & Bivalve cast & High school & 13 & 14 \\
\hline
\end{tabular}

have reviewed the literature on metacarpal fractures in athletes. These authors are comfortable allowing athletes to return to play within 2 weeks following surgery and wound healing. They also recommend 6 weeks of protective equipment to be worn during practice and game competition following fixation [3].

The results of the current study compare favorably with these prior studies in regards to a return to play timeline. In-season athletes in this cohort returned to play on average 7 days before previously reported cohorts. In addition, protective equipment was used an average of 21 days, which is 3 weeks shorter than previous recommendations [3]. Despite the decrease in return to play and brace time, there were no postoperative hardware failure or wound complications. This suggests that treating surgeons could potentially advance return to play protocols and shorten brace duration in metacarpal fractures in football players.

There were several limitations of the present study. It is a retrospective review with a small sample size, which could have introduced biases to the study. The reliance on patient interviews also increases the likelihood of recall bias. The average follow-up was 3 months, and longer follow-up would also better assess any late fracture complications. There was also variability in the surgical treatment performed as well as the type of protective equipment, if any, used after surgery. Nevertheless, the authors believe that the outcomes are valuable in guiding treating surgeons on postoperative management of metacarpal fractures in football players. In addition, there was no comparison treatment group involving nonoperative treatment because the purpose of the study is to evaluate return to play after surgical fixation of metacarpal fractures. Patients were selected for surgery based on standard indications, and athletes that were not indicated for surgical treatment were excluded from the study and were immobilized for 4 to 6 weeks. They were held out of play until union was identified on radiographs. The aim of this paper is not to provide an additional indication for surgery based on early return to athletics, but rather to evaluate the return to play timeline after surgical fixation is performed.

The decision to return to play for athletes is multifactorial and requires a collaborative decision from the surgeon, athlete, and coaching and training staff. Conservative management of metacarpal fractures is a proven and reliable treatment. Data from the present study provides support that when indicated, surgical treatment of displaced metacarpal shaft fractures can result in efficient return to play in football players. Surgical interventions with a plate, nail, or with lag screws prevented fracture displacement and allowed early range of motion postoperatively. There were no re-fractures or complications. Further studies are required examining return to play timelines following metacarpal fracture in this subset of athlete patients.

Conflict of Interest BEE declares that he has no conflict of interest. AJS declares that he has no conflict of interest. DDT declares that he has no conflict of interest. KTA declares that he has no conflict of interest. BAE declares that he has no conflict of interest. JRD declares that he has no conflict of interest. ELC declares that he has no conflict of interest.

Statement of Informed Consent Informed consent was obtained from all patients included within the study.

Statement of Human and Animal Rights All study procedures were followed in accordance with the ethical standards of the responsible committee on human experimentation (institutional and national) and with the Helsinki Declaration of 1975, as revised in 2000 and 2008.

Funding No external funding was required for this study. 


\section{References}

1. Chung KC, Spilson SV. The frequency and epidemiology of hand and forearm fractures in the United States. J Hand Surg [Am]. 2001;26:908-15.

2. Emmett JE, Breck LW. A review and analysis of 11,000 fractures seen in a private practice of orthopedic surgery, 1937-1965. J Bone Joint Surg Am. 1958;40:1169-75.

3. Fufa DT, Goldfarb CA. Fractures of the thumb and finger metacarpals in athletes. Hand Clin. 2012;3:379-88.

4. Geissler WB. Operative fixation of metacarpal and phalangeal fractures in athletes. Hand Clin. 2009;25:409-21.

5. Geissler WB, McCraney WO. Operative management of metacarpal fractures. In: Ring DC, Cohen MS, editors. Fractures of the hand and wrist. New York: Informa Healthcare USA, Inc; 2007. p. 75-90.

6. Giddins GE. The non-operative management of hand fractures. J Hand Surg Eur Vol. 2015;40:33-41.
7. Hardy MA. Principles of metacarpal and phalangeal fracture management: a review of rehabilitation concepts. J Orthop Sports Phys Ther. 2004;34:781-99.

8. Henry MH. Fractures and dislocations of the hand. In: Rockwood CA, Bucholz RW, Court-Brown C, editors. Rockwood and green's fractures in adults. Philadelphia: Lippincott Williams \& Wilkins; 2006. p. 772-855.

9. Khan A, Giddins G. The outcome of conservative treatment of spiral metacarpal fractures and the role of the deep transverse metacarpal ligaments in stabilizing these injuries. J Hand Surg Eur Vol. 2015;40:59-62.

10. Rettig AC, Ramon R, Shelbourne KD, McCarroll JR, Johnson Jr F, Ahlfeld SK. Metacarpal fractures in the athlete. Am J Sports Med. 1989;17:567-72.

11. Strauch RJ, Rosenwasser MP, Lunt JG. Metacarpal shaft fractures: the effect of shortening on the extensor tendon mechanism. J Hand Surg [Am]. 1998;23:519-23.

12. Swenson DM, Yard EE, Collins CL, Fields SK, Comstock RD. Epidemiology of US high school sports-related fractures, 20052009. Clin J Sport Med. 2010;20:293-9. 